\title{
Klinische Forschung am nichtuniversitären Spital
}

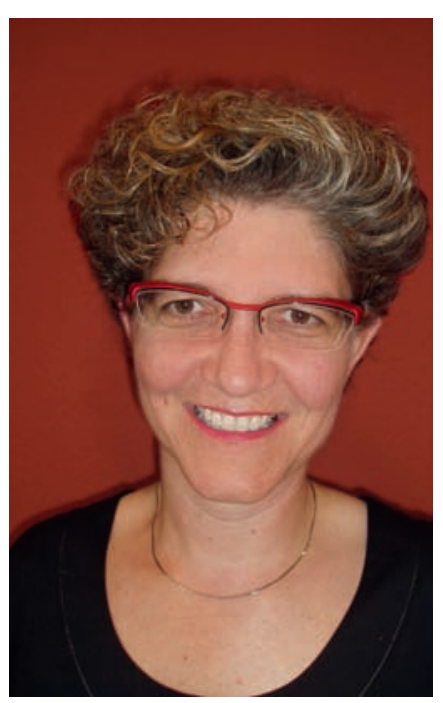

Mit der Sequenzierung des menschlichen Genoms wurde eine grundlegend neue Basis für das Verständnis menschlicher Krankheiten gelegt. Doch wenn die Kenntnis der menschlichen Genomsequenz im klinischen Alltag zu einer generell verbesserten Behandlung von Patienten führen soll, sind nicht nur die biomedizinischen Grundlagenforscher gefordert. Die Erwartungen wurden hoch gesteckt, aber bis heute nur in den wenigsten Fällen erfüllt. Rasch wurden «bottlenecks» gesucht und gefunden, die die Umsetzung dieses molekular-medizinischen Wissens in gute, validierte klinische Konzepte verzögerten. Einer dieser Engpässe ist eine fehlende breitabgestützte klinische Forschungskultur.

Derzeit unterstützen öffentliche Forschungsförderinstitutionen weltweit Programme zur Revitalisierung und zum Ausbau der klinischen Forschung. Das 7. Rahmenprogramm für Forschung und Technologieentwicklung der Europäischen Union [1], das Hauptförderinstrument der Forschung in Europa, widmet beispielsweise einen ganzen Themenkreis der Gesundheit. Es heisst in der Einleitung des Ausschreibungstextes: «Das Hauptziel des Themas «Gesundheit〉 ist es, die Gesundheit der Europäischen Bürger zu verbessern [...]. Dieses Ziel soll in erster Linie mit translationaler Forschung (d.h. mit der Übersetzung von Entdeckungen der Grundlagenforschung in klinische Applikationen) [...] erreicht werden.»

Um die klinische Forschung in der Schweiz zu fördern, hat der Schweizerische Nationalfonds ein Programm initiiert, mit dem er seit 2007 den Aufbau von klinischen Studienzentren (Clinical Trial Units, CTU) unterstützt [2]. Solche CTU sind seither an allen Schweizer Universitätsspitälern und am Kantonsspital St. Gallen eingerichtet worden. Sie bündeln Fachwissen (z. B. in Epide- miologie, Statistik, Pharmakologie) zur Durchführung klinischer Studien und stellen dieses Knowhow den Forschern zur Verfügung. Sie stellen die Verbindung zu den Behörden und Ethikkommissionen her, um den Ablauf von klinischen Studien sicher und reibungslos zu gestalten. Eigentliche Synergien zwischen diesen Zentren verspricht man sich durch eine effiziente Vernetzung in der Swiss Clinical Trial Organisation. Sie ermöglicht die Entwicklung von Standards zur Harmonisierung der Abläufe, die Nutzung gemeinsamer Informationskanäle, die Erarbeitung aufeinander abgestimmter Schulungsmodule oder IT-basierter Datamanagementsysteme, um nur einige Beispiele zu nennen. Nicht nur die Clinical Trial Units untereinander, sondern auch die nichtuniversitären Spitäler der Schweiz sollen in ein solches schweizerisches Studiennetzwerk integriert werden.

Innovation entsteht in der Medizin immer nur durch die aktive Beteiligung an klinischer Forschung. Aktive Beteiligung spannt den Bogen von der Durchführung einer Studie im Auftrag der Pharmaindustrie bis zur Umsetzung der Idee in Form eines eigenen, auch selbstfinanzierten Forschungsprotokolls! Innovation steht in den nächsten Jahren nicht nur im Bereich der Spitzenmedizin, sondern ganz besonders auch im Bereich der Grundversorgung an. Es ist wichtig, dass Schweizer Spitäler diese Innovation nicht nur nachvollziehen, sondern für ihre Patienten mitprägen.

Barbara Biedermann *
Literatur
1 http://cordis.europa.eu/fp7/home_en.html.
2 www.snf.ch/E/funding/projects/ctu/Pages/ default.aspx.

* PD Dr. med. Barbara Biedermann ist Leitende Ärztin für Molekulare Innere Medizin am Kantonsspital Bruderholz BL und setzt sich für die Anliegen der nichtuniversitären Spitäler beim Aufbau der Swiss Clinical Trial Organisation ein.

\begin{abstract}
«Klinische Forschung am nichtuniversitären Spital» ist Thema eines ganztägigen Workshops, der am 16. Oktober 2008 am Bruderholzspital BL stattfindet. Der Workshop zeigt einen auch für nichtuniversitäre Spitäler realistischen, gangbaren Weg zur erfolgreichen, unabhängigen Studientätigkeit auf. Ein Strategiemeeting lädt die Teilnehmer ein, mittel- und langfristige Ziele eines schweizerischen Studiennetzwerks im Bereich der Grundversorgung festzulegen. Ob dies nun die Prüfung eines neuen Medikamentes bei Arteriosklerose oder Begleitforschung zur DRG-Einführung ist: Spitäler der Grundversorgung sollen mitbestimmen, was erforscht werden soll! Weitere Auskünfte erteilt PD Dr. med. Barbara C. Biedermann, Medizinische Universitätsklinik, Kantonsspital Bruderholz, 4101 Bruderholz, Tel. 0614363144 , E-Mail: barbara.biedermann@unibas.ch.
\end{abstract}

